Pediat. Res. 2: 85-93 (1968)

Ghondrogenesis cortisol mesenchymal cells myogenesis teratogenesis

\title{
Cortisol-Induced Malformation in an In Vitro Developmental Model
}

\author{
R. UMANSKY ${ }^{[15]}$ \\ Department of Pediatrics, Stanford University School of Medicine, \\ and Stanford Children's Convalescent Hospital, Palo Alto, California, USA
}

Extract

Morphologic effects of cortisol upon development were studied in an in vitro model in which populations of free mesenchymal cells, derived from embryonic mouse limb bud, form a heterogeneous tissue, and in five to six days develop characteristic cartilaginous and muscular elements (fig. 2). Development takes place in microwells with bases of a cellulose ester filter.

Cortisol was incorporated into the culture medium at the concentrations shown in table I at various stages in culture (first to fourth day) and for variable periods of time (two hours to six days). The initial cell population density on the filter membrane was varied (table II).

Cortisol concentrations of $1.4 \times 10^{-5} \mathrm{M}$ and $7.0 \times 10^{-5} \mathrm{M}$ for up to six days had no visible effects on formation of a tissue syncytium or subsequent differentiation. Concentrations of $1.1 \times 10^{-3} \mathrm{M}$. for longer than 48 hours led to death of the bulk of cells. Intermediate concentrations or shorter exposures were compatible with tissue viability but were associated with morphologic abnormalities. These abnormalities largely resulted from interference with cell aggregation, chondrogenesis and myogenesis.

The formation of compact cellular aggregates was impaired by exposure to cortisol concentrations between $1.4 \times 10^{-4} \mathrm{M}$ and $1.1 \times 10^{-3} \mathrm{M}$. Varying degrees of inhibition were seen, extending from moderate limitation of compact aggregate size, alteration in shape and loss of distinct aggregate margins to total substitution of loose clusters of cells for compact aggregates. The severity of effects was directly related to cortisol concentration and duration of exposure and inversely related to initial cell population density (table III). Later stages in the development of compact aggregates were inhibited when exposure began at some time after the first day. Neither inhibition of cell movement on the filter surface nor restriction in cell population by cortisol appeared to be major factors interfering with aggregation. When exposure was discontinued prior to three days, compact aggregation resumed.

Chondrogenesis was totally inhibited by cortisol concentrations of $1.1 \times 10^{-3} \mathrm{M}$ for more than one day and partially inhibited by concentrations of $7.0 \times 10^{-4} \mathrm{M}$ and $3.5 \times 10^{-4} \mathrm{M}$. In the latter instances, the effects varied with stage of exposure. Exposures for the first two or three days of culture resulted in fewer cartilage centers, confined mainly to the membrane center. Similar exposures during the last three days of culture restricted the size of cartilage centers but did not affect distribution on the filter surface; such exposures were associated with diminished intercellular metachromasia upon staining with toluidine blue. With exposure for the entire culture period, chondrogenesis was uncommon even at such lower concentrations.

Myogenesis was inhibited at concentrations of $2.0 \times 10^{-4} \mathrm{M}$ or greater. Exposure in the first two days of culture impaired bipolar transformation of myoblasts. Exposure at a later time inhibited multinucleated myoblast and myotube development and restricted the number of myogenic cells. 


\section{Speculation}

The present investigation demonstrates that structural anomalies can be produced by a glucocorticoid in an in vitro developmental model and their evolution observed. The model is suitable for studying features of abnormal development because of the simplified morphogenesis inherent in the system. It offers opportunities for varying not only the conditions of exposure but also the cell source. The degree of relevance of the model to the in vivo situation, however, remains to be identified.

\section{Introduction}

The complex nature of growth, differentiation and morphogenesis in the embryo makes difficult not only the study of that aberrant cell function which leads to teratogenesis but also the understanding of those mechanisms by which these effects are translated into gross structural anomalies. In an effort to simplify the problem, an in vitro model has been employed and its usefulness examined. In this model, a tissue with characteristic cartilage, muscle and connective tissue elements develops from mesenchymal cells [11]. Attention was directed at two questions. Can characteristic patterns of malformation be induced in this simplified in vitro system by introduction of an agent which interferes with normal development? If so, can the processes which result in such malformations be observed and analyzed?

The agent used was cortisol. Glucocorticoids rarely if ever cause malformations in man $[2,10]$; however, at nonphysiologic concentrations, they are potent teratogens and inhibitors of growth in several experimental animals $[5,6,7,9]$, affecting a variety of tissue types at various embryonic stages. Prominent target tissues are those of mesenchymal origin.

\section{Materials and Methods}

A full description of the tissue source, the culture techniques and subsequent in vitro development has been given in a previous paper [11]. Embryonic forelimb mesenchyme of $\mathrm{C}_{3} \mathrm{H}$ mice at 11 days of gestation was used. At this stage, neither chondrogenic preskeletal cell condensations nor myoblasts are seen. The mesenchyme was dissociated into its component cells employing calcium- and magnesium-free solutions, proteolytic enzymes (trypsin and pancreatin) and mechanical fragmentation and shearing. Aliquots of the resultant cell suspension, adjusted with medium ${ }^{1}$ to the concentrations desired, were delivered to the center of microwell assemblies [1] with round cellulose ester filter bases (fig. 1). Under these conditions, the cells settling onto the filter were distributed randomly except that there was a gradual decrease in cell popula-

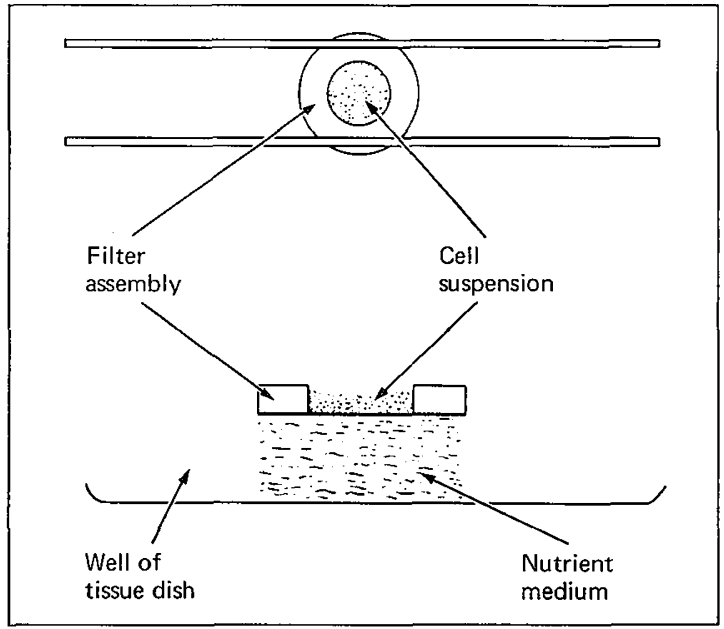

Fig.1. Diagram of filter microwell assembly (AuerBACH and Grobstein [1]).

tion density from center to periphery. Cultures, incubated at $37^{\circ}$ in a $5 \% \mathrm{CO}_{2}$, high humidity environment, were maintained for periods up to six days. Periodic observations in the live and fixed (Bouin's solution) state were made using light and phase microscopy. The presence of toluidine blue $0.1 \%$ in $30 \%$ ethanol) intercellular metachromasia indicated the presence of acid mucopolysaccharides of cartilage.

The cultures were exposed to cortisol (Upjohn Sterile Aqueous Cortef $)^{2}$ in the medium. Initially, cortisol was incorporated into the medium at the start

1 The medium is a complex one, consisting of $85 \%$ Eagle's basal medium with Earle's balanced salt solution; $10 \%$ horse serum; $3 \%$ chick embryo extract; $1 \%$ L-glutamine; and supplements of penicillin G $(22,500$ units), streptomycin sulfate $(5 \mathrm{mgm})$ and nystatin (100 units) per $\mathrm{ml}$ serum.

2 Each $\mathrm{ml}$ of this preparation contains cortisol, $15 \mathrm{mg}$; sodium carboxymethylcellulose, low viscosity, $4.8 \mathrm{mg}$; sodium chloride, $8.7 \mathrm{mg}$; polysorbate (Tween 80 ), $3.85 \mathrm{mg}$; benzyl alcohol, $8.7 \mathrm{mg}$; water for injection, q. s. 
of culture for one day at concentrations ranging from $1.4 \times 10^{-5} \mathrm{M}$ to $1.1 \times 10^{-3} \mathrm{M}$, the upper limit of solubility. Concentrations in the range of $10^{-4} \mathrm{M}$ to $10^{-3} \mathrm{M}$ are estimated to be of the same order of magnitude as those occurring in the extracellular fluid of mice when cortisol is used to induce cleft palate in utero [8]. Based on preliminary results, a more restricted range of concentrations $\left(1.4 \times 10^{-4} \mathrm{M}\right.$ to $\left.1.1 \times 10^{-3} \mathrm{M}\right)$ was selected for detailed study. The age of the culture at which exposure was begun (first, second, third or fourth day), the duration of exposure (two hours, six hours, one day, two days, three days, six days) and the initial cell population density $\left(2 \times 10^{3}\right.$ to $2.5 \times 10^{4}$ cells $\left./ \mathrm{mm}^{2}\right)$ were varied. Table I provides details on the number of cultures studied at various cortisol concentrations. In the

Table $I$. Number of cultures exposed to cortisol at various concentrations

\begin{tabular}{lc}
\hline Cortisol concentration & $\begin{array}{l}\text { Number of } \\
\text { cultures }\end{array}$ \\
\hline $1.1 \times 10^{-3} \mathrm{M}$ & 34 \\
$7.0 \times 10^{-4} \mathrm{M}$ & 61 \\
$3.5 \times 10^{-4} \mathrm{M}$ & 19 \\
$2.0 \times 10^{-4} \mathrm{M}$ & 4 \\
$1.4 \times 10^{-4} \mathrm{M}$ & 13 \\
$7.0 \times 10^{-5} \mathrm{M}$ & 4 \\
$1.4 \times 10^{-5} \mathrm{M}$ & 4 \\
& \\
\hline
\end{tabular}

Table II. Initial cell population density of cortisoltreated and medium-control cultures

\begin{tabular}{llc}
\hline $\begin{array}{l}\text { Initial cell population } \\
\text { density (cells } / \mathrm{mm}^{2} \text { at } \\
\text { membrane center) }\end{array}$ & $\begin{array}{l}\text { Number of } \\
\text { cortisol- } \\
\text { treated } \\
\text { cultures }\end{array}$ & $\begin{array}{l}\text { Number of } \\
\text { controls }\end{array}$ \\
\hline 2,000 & 8 & 7 \\
3,000 & 10 & 8 \\
4,000 & 18 & 4 \\
5,000 & 23 & 27 \\
6,000 & 7 & 4 \\
7,000 & 2 & 1 \\
8,000 & 9 & 4 \\
9,000 & 11 & 4 \\
10,000 & 19 & 17 \\
12,000 & 15 & 10 \\
15,000 & 11 & 13 \\
20,000 & 2 & 1 \\
25,000 & 4 & 4 \\
\hline
\end{tabular}

amount employed, the cortisol vehicle was shown to have no effect on growth characteristics of the tissue. The results are based on 139 cortisol-treated cultures and 104 medium-control cultures studied in 29 experiments. Control cultures were identical in all constituents to cortisol cultures except for absence of the glucocorticoid. Cortisol and control preparations, matched for initial cell population density, were cultured simultaneously in each experiment (table II).

\section{Results}

\section{Development in Controls}

In culture, cytodifferentiation paralleled that seen in the early phases of in vivo limb bud mesenchyme development, but morphogenesis was much simplified. The sequence of normal in vivo development is illustrated in figure 2.

The cells of the dissociated mesenchyme distributed on the filter surface are mobile. The first indication of compact aggregation is the formation of clusters of loosely arranged cells in the first few hours in culture. In the first day, these develop into aggregates of adherent, tightly packed rounded cells. On the second and third day, fusion of compact aggregates and proliferative activity results in larger but fewer aggregates. At the same time, small, widely separated aggregates are often unable to fuse and lose their compact character entirely by spreading, blending with the proliferating monolayer. These latter changes are more marked at the periphery of the filter surface.

Only aggregates which retain substantial compact cellular cores subsequently undergo chondrogenesis. This begins in the interior of compact aggregates on the second or third day and extends outward. By the sixth day, cartilage mounds and ridges composed of tridimensional whorls of concentric cells embedded in matrix have formed.

Around developing cartilage elements, the surrounding monolayer which develops along fibroblastic lines serves as a substrate for muscle differentiation. At the first day, the presumptive muscle cells form small groups of dark-staining, loosely associated polyhedral cells on the monolayer surface. Subsequently they undergo bipolar transformation, proliferate and form longitudinally arranged multinucleated myoblasts and occasional myotubes.

Although the duplication of morphology characteristic of in vivo development does not occur from culture to culture, certain aspects of development are replicable. The size, number and general distribution of compact aggregates and, later on, cartilage and muscle elements are closely related to initial cell population density. Because of the central-peripheral gradient in 


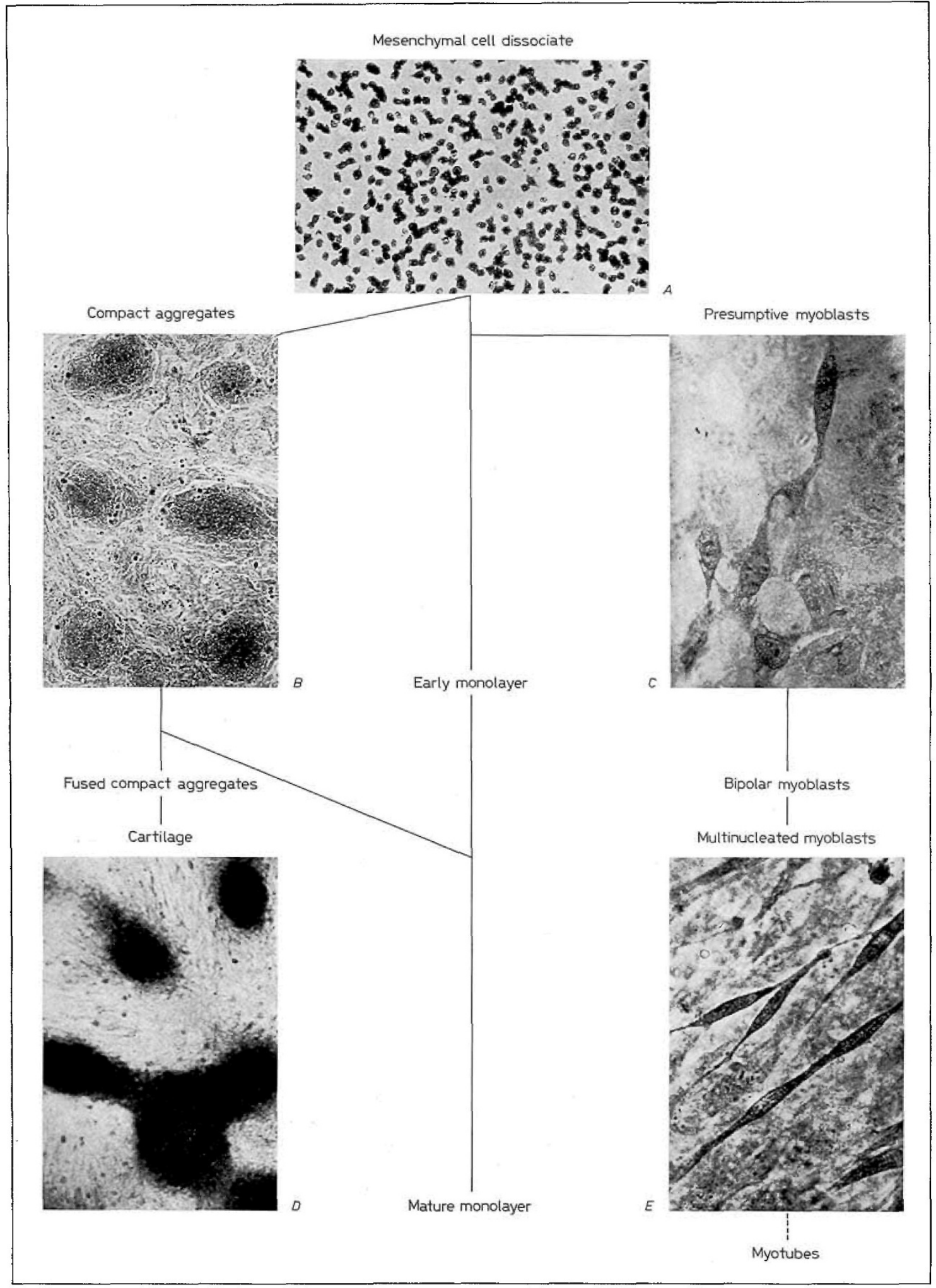


initial cell population, development at the membrane center is usually different from that of the periphery. Thus, at one day in culture, the center contains compact aggregates which are larger than those at the periphery, and atsix days, cartilage masses are more numerous, more varied in shape and often larger at the center.

Effects of Cortisol

Exposure to relatively low concentrations of cortisol $\left(1.4 \times 10^{-5} \mathrm{M}, 7.0 \times 10^{-5} \mathrm{M}\right)$ for up to six days had no visible effect in any of the developing cultures. Exposures to high concentrations $\left(1.1 \times 10^{-3} \mathrm{M}\right)$ for periods longer than 48 hours invariably led to death of the bulk of cells in culture. However, when cortisol was used either at intermediate concentrations or for shorter periods, overall restriction in cell population was much less pronounced and a viable morphologically abnormal tissue formed. A range of reproducible malformation was seen, varying with dose, time and duration of exposure, as well as with initial cell population density. Certain developmental processes were characteristically affected.

Compact aggregation. Cortisol incorporated into the medium at the start of culture for one day at concentrations from $1.4 \times 10^{-4} \mathrm{M}$ through $1.1 \times 10^{-3} \mathrm{M}$ always interfered with the organization of compact aggregates. Inhibition was indicated by a decrease in aggregate compactness and size; cortisol did not abolish cell movement on the filter surface since cells retained the capacity to cluster. The severity of the changes varied with the cortisol level within this range, the initial cell population density and the duration of exposure.

At the highest concentrations, loose clusters of cells formed which lacked the tightly packed organization of controls (fig. 3). There were occasional gaps between cells and overlapping of cell borders. At lower concentrations, lesser degrees of disorganization of compact aggregation were seen. In such instances, compact aggregates formed but were smaller and had less welldefined margins. Many were less rounded than normal. Compactly arranged cells in culture were fewer. At given concentrations of cortisol, variations in response

Fig.2. Diagram of in vitro tissue reconstruction from mesenchymal cell dissociate and cartilage and muscle histogenesis. A. Dissociated cells, seen one hour after delivery, reconstitute a tissue fabric on the filter surface $(64 \times)$. B. Compact cellular aggregates connected by a monolayer sheet or web develop $(64 \times)$. C. At the same time presumptive myoblasts appear on the monolayer $(400 \times) . D$. The compact aggregates form characteristic cartilage elements $(64 \times)$. E. Muscle elements develop on the monolayer surface $(400 \times)$. Whole mounts. Toluidine Blue. Phase.
Table III. Inhibition of compact aggregation by cortisol during first day of culture as related to initial cell population density

\begin{tabular}{llll}
\hline $\begin{array}{l}\text { Cortisol } \\
\text { concentration }\end{array}$ & \multicolumn{3}{c}{$\begin{array}{c}\text { Initial cell population density } \\
\left.\text { (cells } / \mathrm{mm}^{2}\right)\end{array}$} \\
\cline { 2 - 4 } & 8 to $12 \times 10^{3}$ & 3 to $5 \times 10^{3}$ & $2 \times 10^{3}$ \\
\hline $1.1 \times 10^{-3} \mathrm{M}$ & ++ & +++ & +++ \\
$7.0 \times 10^{-4} \mathrm{M}$ & + & ++ & +++ \\
$1.4 \times 10^{-4} \mathrm{M}$ & - & + & ++ \\
\hline
\end{tabular}

+++ Glusters of loosely associated cells.

++ Compact aggregates smaller than normal and often less rounded; margins less well demarkated.

+ Slight diminution in size of compact aggregates; often less rounded.

- No deviation from normal.

could be related to initial cell population density, the effects being more severe the lower the population density (table III). Inhibition of aggregation could be demonstrated following exposure for periods as short as two hours, although longer exposures led to more severe effects.

Later stages of compact aggregate development, such as fusion, were impaired when exposure to cortisol was prolonged beyond the first day or begun after this point. Exposure for two or three days led to more marked antiaggregation effects than exposure for one day at similar cortisol levels and initial cell population densities. Distinctions in aggregate size between cortisol-treated and control cultures were often greater at three days. Such effects were more marked at the periphery where initial cell population density was lower (fig.4). When exposure took place after the first day, the larger aggregates in culture persisted but did not enlarge further as did controls.

When cortisol-treated and control cultures with approximately similar cell populations were compared at various times in culture, compact aggregation was very much superior in controls. This indicated that restriction in cell population by cortisol was a relatively insignificant factor in limiting compact aggregate development.

With removal of cortisol from the medium, compact aggregation resumed if exposure was for a period of less than three days' duration (fig. 5) ; however, development of control aggregates remained more advanced.

Chondrogenesis. Cortisol in high concentrations inhibited chondrogenesis. No cartilage developed after exposure to concentrations of $1.1 \times 10^{-3} \mathrm{M}$ at any stage of 


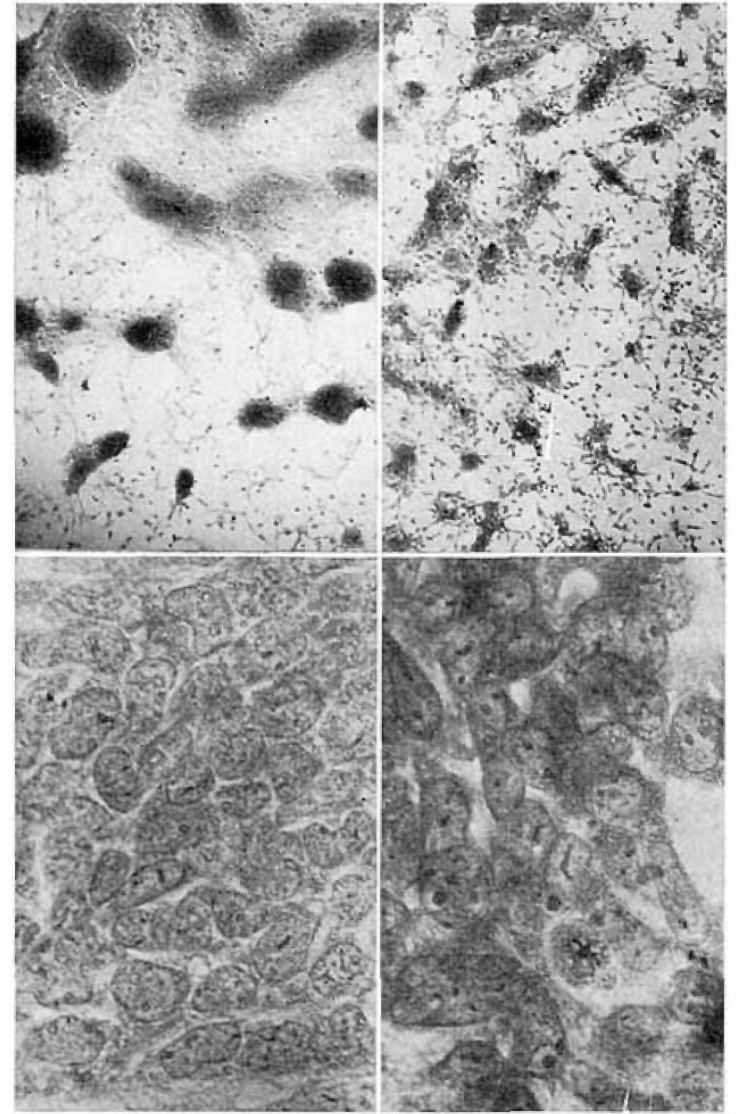

Fig.3. Low upper) and high (lower) magnification views of inhibition of compact aggregation by cortisol $\left(1.1 \times 10^{-3} \mathrm{M}\right)$ in a 20 -hour-old culture. Controls at left. Tightly packed organization is lacking in cortisol cultures. Low power views show effect of cell population gradient. At high magnifications, intercellular gaps and uneven overlapping of cells are evident. Whole mounts. Toluidine Blue $(33 \times),(525 \times)$. Phase.

culture when maintained for periods longer than one day. Somewhat lower concentrations $\left(7.0 \times 10^{-4} \mathrm{M}\right.$, $3.5 \times 10^{-4} \mathrm{M}$ ) for periods of two to three days permitted modified types of cartilage formation. The morphologic findings differed, however, depending on whether cortisol was incorporated into the medium early or late (fig. 6). When cortisol was used for the first two to three days, in comparison with the normal, fewer cartilage elements were seen at five or six days. These were largely confined to the membrane center. When compared with controls, intercellular metachromatic staining was not reduced. Cartilage elements were slightly

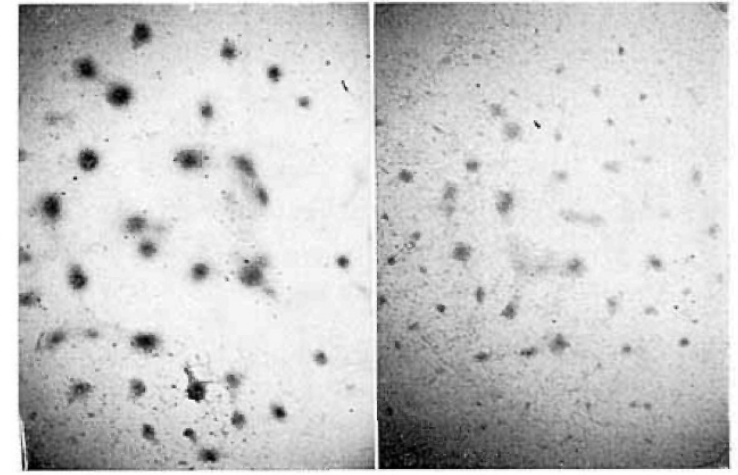

Fig.4. Low magnification views $(13 \times)$ of cortisol restriction of compact aggregatesize, particularly marked at periphery. Partially inhibitory concentrations of cortisol $\left(7.0 \times 10^{-1} \mathrm{M}\right)$ for three days. Control at left. No cartilage is forming in cortisol culture. Whole mounts. Toluidine Blue. Light.

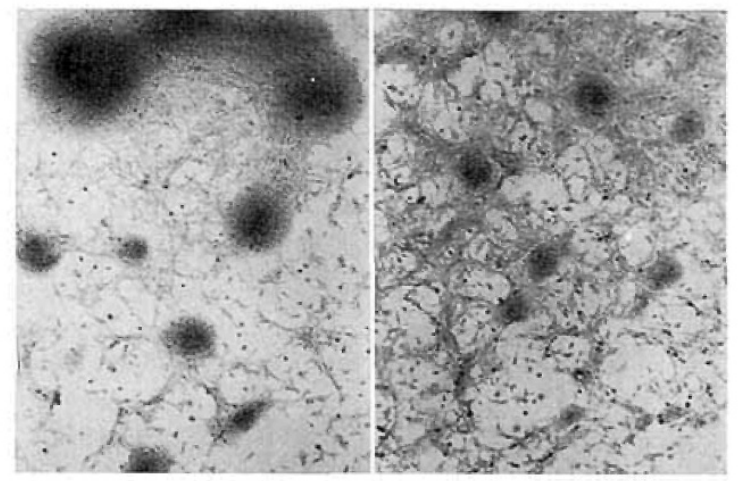

Fig.5. Compact aggregates have formed at 48 hours following removal of cortisol $\left(1.1 \times 10^{-3} \mathrm{M}\right)$ after the first 24 hours. Control at left. Development lags behind control where aggregates tend to be larger and fusion is more prominent. Also compare with fig. 3. Whole mounts. Toluidine Blue $(33 \times)$. Phase.

smaller than normal. On the other hand, when cortisol was incorporated in the medium on the fourth day and maintained for two to three days, the development of cartilage from fused compact aggregates was impaired. Cartilage elements appeared normally distributed and numerous, but were much smaller than those in the control. A lesser degree of intercellular metachromasia was seen. When cortisol was present in the medium for five or six days at these levels, cartilage developed less frequently. When it appeared, its distribution resembled the pattern associated with exposure during the first half of culture. 

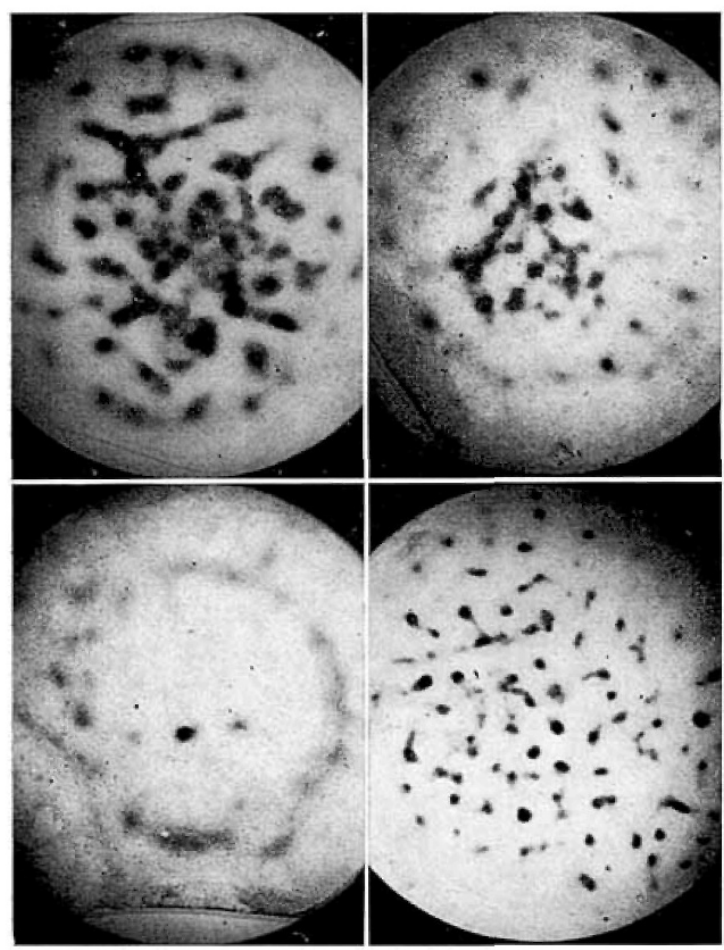

Fig.6. Low magnification views $(13 x)$ at $5 \frac{1}{2}$ days showing inhibition of chondrogenesis by cortisol as related to stage of exposure and concentration. In control at upper left, cartilage is seen both at the center and towards the periphery. At upper right where cortisol was employed for the first 3 days at partially inhibitory concentrations $\left(3.5 \times 10^{-4} \mathrm{M}\right)$, cartilage is largely confined to central regions. At the lower left where double the dose of cortisol was used $\left(7.0 \times 10^{-4} \mathrm{M}\right)$ during the same period, effects are more pronounced. With cortisol exposure $\left(7.0 \times 10^{-4} \mathrm{M}\right)$ for the last $2 \frac{1}{2}$ days (lower right) cartilage elements occur both centrally and peripherally, but are much smaller than normal. Whole mounts. Toluidine Blue. Light.

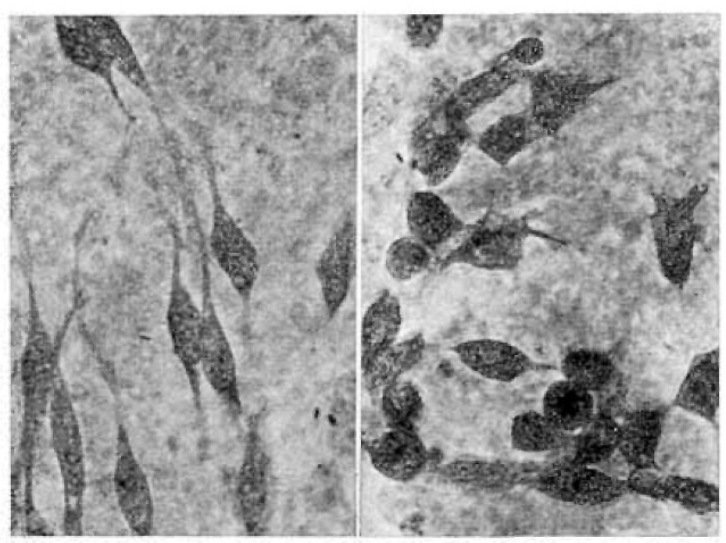

Myogenesis. Cortisol interfered with the development of muscle at concentrations of $2.0 \times 10^{-4}$ or greater. The severity of the effects varied with the duration of exposure and concentration. Following exposure early in culture, presumptive myoblasts were still seen on the monolayer surface in normal numbers, but their bipolar transformation was strikingly impaired (fig. 7). The cell bodies remained plump instead of elongated. Cytoplasmic processes linking myogenic cells were infrequent and the cells were in loose clumps. Removal of cortisol from the medium after the first or second day permitted renewed muscle differentiation. If cortisol was incorporated into the medium at this point or maintained for longer periods, fewer myogenic cells were present and the appearance of multinucleate myoblasts and myotubes in culture was inhibited (fig. 8). Somewhat similar but less severe changes were seen when cortisol was first incorporated into the medium after the second day.

At all cortisol concentrations less than $1.1 \times 10^{-3} \mathrm{M}$, the monolayer developed; however, the possibility of changes in its structure with cortisol has not yet been investigated.

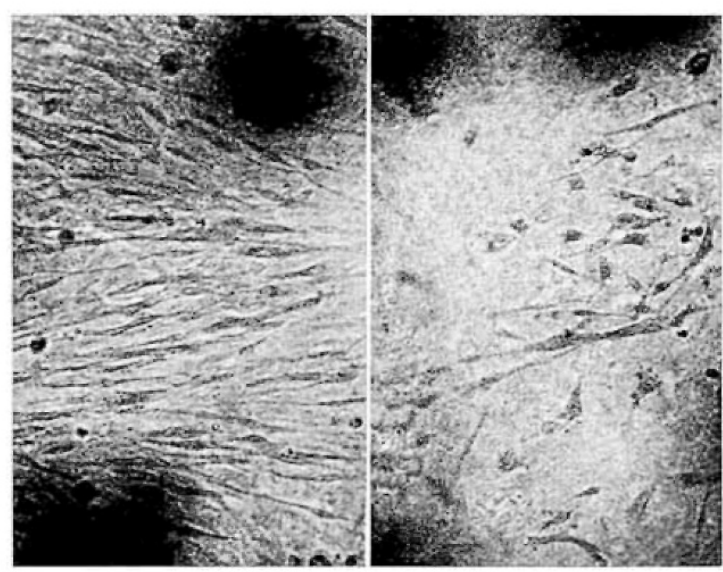

Fig.8. Inhibition of multinucleate myoblast development and restriction in myoblast number by relatively low concentrations of cortisol $\left(2.0 \times 10^{-4} \mathrm{M}\right)$ present for 6 days in culture. Cartilage development is relatively normal. Control at left. Whole mount. Toluidine Blue $(130 \times)$. Phase.

Fig.7. High magnification views $(525 \times)$ of inhibition of bipolar transformation of presumptive myoblasts by cortisol $\left(7.0 \times 10^{-4} \mathrm{M}\right)$ present for first 2 days. Control at left. In cells exposed to cortisol cytoplasmic processes linking cells are less evident. Whole mounts. Toluidine Blue. Phase. 
Discussion

Important parallels exist between cytodifferentiative processes in the in vitro model and early in embryonic limb mesenchymal development. Myogenesis is similar up to the myotube stage. Cartilage-forming compact aggregates in culture and chondrogenic limb bud condensations [13] are derived from the same tissue source and are histologically similar. Their cells differ from most other types of mesenchyme in their rounded form and close association.

Nevertheless, in vitro effects of cortisol described in this report cannot be directly extrapolated to specific teratologic consequences of this drug in animals. As an example, the development of the aggregated state necessary for chondrogenesis [3] is inhibited by cortisol in this model. The spectrum of changes observed strongly suggests that it has an adverse effect on the adhesiveness of cells necessary for close intercellular association [4]. However, the initial stages of compact aggregation in culture are different from those seen in embryonic mesenchymal condensation. In culture, cells at first act as individuals since the preexisting tissue organization has been totally disrupted and a multicellular structure must form anew. In developing condensations in vivo, it is more likely that cells retain some of their tissue connections and that localized cell proliferation is involved. Thus, the relevance of these in vitro findings to established teratogenic effects of glucocorticoids on in vivo skeletogenic condensation [9] requires further study.

The simplified development characteristic of the in vitro model aids morphogenetic investigation. The developing cartilaginous and muscular elements are simpler in form and their anatomic relations less complex than those seen in vivo. This facilitates analysis of contrasting patterns of cortisol-induced malformation. For instance, partially suppressive doses present early in culture act regionally, permitting subsequent chondrogenesis centrally but inhibiting it peripherally. Later, however, cortisol has a more generalized effect; the distribution of cartilage elements on the filter surface is not markedly altered, although reduction in size is pronounced. The former effect is largely a consequence of inhibition of aggregation. Superimposed on the initial radial population gradient, cortisol limits the size of the compact aggregate until, finally, the aggregate at the periphery becomes too minute to support chondrogenesis. The latter effect was associated with reduced intercellular metachromatic staining in cartilage, suggesting reduced synthesis of acid mucopolysaccharide. High concentrations of cortisol are known to inhibit mucopolysaccharide sulfation [12], an effect which would not be expected to be limited to any particular portion of the filter membrane.
Summary

Characteristic effects ranging from malformation to death were seen when nonphysiologic concentrations of cortisol were used in an in vitro developmental model in which free limb bud mesenchymal cells were cultured to form heterogeneous structures, which later differentiated into a tissue with distinctive cartilage and muscle elements. The malformative effects of cortisol varied with concentration, stage of development at time of application and duration in culture, as well as with initial cell population density. A partially reversible interference with the formation of compact prechondrogenic mesenchymal aggregates was demonstrated. Cortisol also interfered with chondrogenes.s and myogenesis.

\section{References and Notes}

1. Auerbach, R. and Grobstein, G.: Inductive interaction of embryonic tissues after dissociation and reaggregation. Exp. Cell Res. 15: 384 (1958).

2. Bonglovanni, A.M. and MaPadden, A.J.: Steroids during pregnancy and possible fetal consequences. Fertil. and Steril. 11: 181 (1960).

3. Cooper, G.W.: Induction of somite chondrogenesis by cartilage and notochord: a correlation between inductive activity and specific phases of cytodifferentiation. Ph.D. Thesis, pp.69-72 (Stanford University, Palo Alto 1963).

4. Gurtis, A.S.G.: Gell contact and adhesion. Biol. Rev. 37: 82 (1962).

5. Farnstar, T.D.: Cortisone-induced congenital cleft palate in rabbits. Endocrinology 55: 502 (1954).

6. HoAr, R.M.: Similarity of congenital malformations produced by hydrocortisone to those produced by adrenalectomy in guinea pigs. Anat. Rec. 144: 155 (1962).

7. KaLter, H. : Factors influencing frequency of cortisone-induced cleft palate in mice. J.exp. Zool. 134: 449 (1957).

8. Kalter, H. and Fraser, F. G. : Production of congenital defects in offspring of pregnant mice treated with compound F. Nature (Lond.) 169: 665 (1952).

9. Moscona, M.H. and Karnofsky, D. A.: Cortisoneinduced modifications in the chick embryo. Endocrinology 66: 533 (1960).

10. Popert, A.J.: Pregnancy and adrenocortical hormones. Brit.med.J. 5283: 967 (1962).

11. Umansky, R.: The effect of cell population density on the developmental fate of reaggregating mouse limb bud mesenchyme. Develop.Biol. 13: 31 (1966). 
12. Whitehouse, M.W. and LAsh, J.W.: Effect of cortisone and related compounds on the biogenesis of cartilage. Nature, Lond. 189: 37 (1961).

13. ZwIlling, E.: Limb morphogenesis. Advanc. Morphogenesis 1: 301 (1961).

14. This work was supported in part by grants from the John A. Hartford Foundation, The National Foun- dation, and by grants AM-05223 and TI-HD-49 from the National Institutes of Health, USPHS. 15. Requests for reprints should be addressed to: Dr. R. Umansky, Department of Pediatrics, Children's Hospital Medical Center of Northern California, 51st and Grove Streets, Oakland, Cal. 94609 (USA). 